\title{
Total Synthesis of Herbertenolide by Stereospecific Formation of Quaternary Centers in a Crystalline Ketone
}

\author{
Danny Ng, Zhe Yang, and Miguel A. Garcia-Garibay* \\ Department of Chemistry and Biochemistry, University of California,Los Angeles, CA 90095-1569
}

Page S2-S5: General Synthetic Procedures and Spectral Data

Page S6: $\quad{ }^{1}$ H NMR Spectrum of trans-2-(2-Methoxy-5-methylphenyl)cyclohexanol (5)

Page S7: $\quad{ }^{1}$ H NMR Spectrum of 2-(2-Methoxy-5-methylphenyl)cyclohexanone (6)

Page S8: $\quad{ }^{1}$ H NMR Spectrum of 2-(2-Methoxy-5-methylphenyl)-2-methylcyclohexanone (6a)

Page S9: $\quad \quad{ }^{1} \mathrm{H}$ NMR Spectrum of 2-(2-Methoxy-5-methylphenyl)-2,6-dimethylcyclohexanone (7)

Page S10: $\quad{ }^{1} \mathrm{H}$ NMR Spectrum of trans-3-(2-Methoxy-5-methylphenyl)-1,3-dimethyl-2-oxocyclohexanecarboxylic acid methyl ester (3)

Page S11: $\quad{ }^{1} \mathrm{H}$ NMR Spectrum of trans-2-(2-Methoxy-5-methylphenyl)-1,2-dimethylcyclopentanecarboxylic acid methyl ester (2)

Page S12: $\quad{ }^{1} \mathrm{H}$ NMR Spectrum of ( \pm )-herbertenolide (1). 


\section{Experimental Section.}

General: Tetrahydrofuran (THF) was distilled over sodium-benzophenone ketyl. Diisopropylamine was distilled over $\mathrm{CaH}_{2}$.

Commercial reagents of the highest purity available were used without further purification. ${ }^{1} \mathrm{H}$ and ${ }^{13} \mathrm{C}$ NMR spectra were obtained with a Bruker ARX500 or Avance500 spectrometer in $\mathrm{CDCl}_{3}$. IR spectra were obtained with a Perkin-Elmer spectrometer either in $\mathrm{KBr}$ disks or as neat oils. High resolution mass spectra were obtained by electron ionization. Melting points were uncorrected. All reactions were carried out in oven-dried glass ware under argon atmosphere.

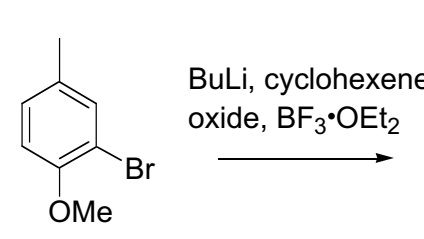

4

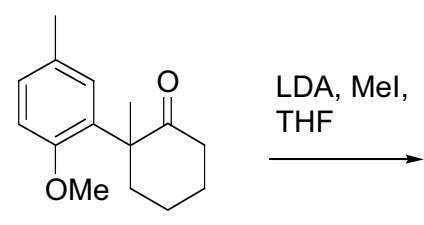

$6 a$

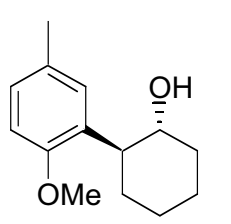

5

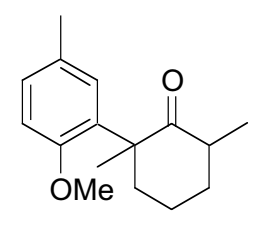

7

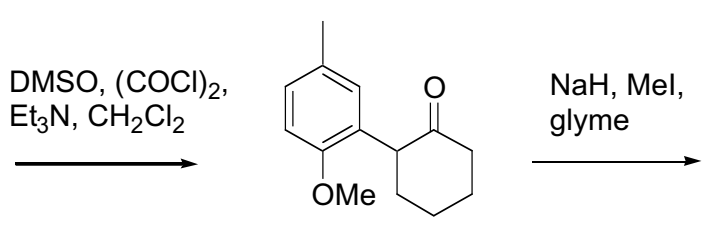

6
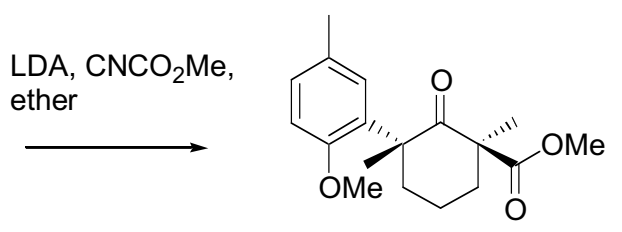

3

hv, solid,

$0^{\circ} \mathrm{C}, \lambda>310 \mathrm{~nm}$

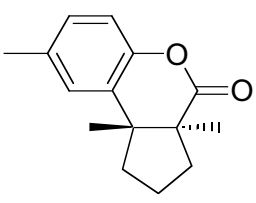

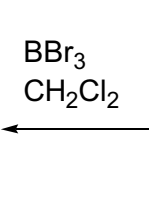

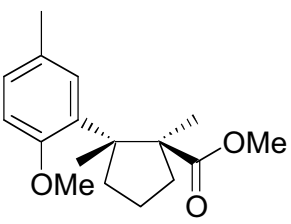

Page S2 
Trans-2-(2-Methoxy-5-methylphenyl)cyclohexanol (5). A $50 \mathrm{~mL}$ round bottom flask equipped with a magnetic stirring bar was charged with 2-bromo-4-methylanisole (4) $(0.735 \mathrm{~mL}, 5.10 \mathrm{mmol})$ and THF $(8 \mathrm{~mL})$. After cooling to $-78^{\circ} \mathrm{C}, n$-butyllithium $(3.25 \mathrm{~mL}$, $5.20 \mathrm{mmol}, 1.6 \mathrm{M}$ in hexanes) was added dropwise. The reaction mixture was stirred at $-78^{\circ} \mathrm{C}$ for $1 \mathrm{~h}$. Cyclohexene oxide $(0.257 \mathrm{~mL}$, $2.55 \mathrm{mmol}$ ) was added, followed immediately by $\mathrm{BF}_{3} \cdot \mathrm{OEt}_{2}(0.485 \mathrm{~mL}, 3.82 \mathrm{mmol})$. After stirring at $-78^{\circ} \mathrm{C}$ for $1.5 \mathrm{~h}$, reaction was quenched with saturated $\mathrm{NaHCO}_{3}$ and warmed to r.t. Product was extracted with ethyl acetate $(2 \times 20 \mathrm{~mL})$. The combined organic layer was washed with cold $10 \% \mathrm{NaOH}$ and brine. After drying over $\mathrm{MgSO}_{4}$, solvent was removed in vacuo. Purification by column chromatography $(15 \% \mathrm{EtOAc} / \mathrm{hexanes})$ afforded cyclohexanol $5(0.546 \mathrm{~g}, 98 \%)$ as a viscous, colorless oil. ${ }^{1} \mathrm{H}$ NMR: $\delta 1.20-1.60(\mathrm{~m}$, $4 \mathrm{H}), 1.70-1.90(\mathrm{~m}, 4 \mathrm{H}), 2.12-2.20(\mathrm{~m}, 1 \mathrm{H}), 2.29(\mathrm{~s}, 3 \mathrm{H}), 2.90-3.00(\mathrm{~m}, 1 \mathrm{H}), 3.71-3.73(\mathrm{~m}, 1 \mathrm{H}), 3.80(\mathrm{~s}, 3 \mathrm{H}), 6.79(\mathrm{~d}, 1 \mathrm{H}, \mathrm{J}=8.3 \mathrm{~Hz})$, $6.99(\mathrm{dd}, 1 \mathrm{H}, \mathrm{J}=8.3 \mathrm{~Hz}, 2.1 \mathrm{~Hz}), 7.05(\mathrm{~d}, 1 \mathrm{H}, \mathrm{J}=2.1 \mathrm{~Hz}) .{ }^{13} \mathrm{C}$ NMR: $\delta$ 20.63, 25.11, 26.16, 32.33, 35.14, 55.64, 73.98, 110.82 , 127.66, 128.02, 130.09, 131.15, 155.62. IR (neat): 3444, 2997, 2930, 2855, 1504, 1463, 1447, 1250, 1233, 1133, 1064, $1037,862,804$ $\mathrm{cm}^{-1}$. EI HRMS: calculated for $\mathrm{C}_{14} \mathrm{H}_{20} \mathrm{O}_{2} 220.1463$, found 220.1462 .

2-(2-Methoxy-5-methylphenyl)cyclohexanone (6). To a $50 \mathrm{~mL}$ round bottom flask equipped with a magnetic stirring bar were added oxalyl chloride $(0.238 \mathrm{~mL}, 2.73 \mathrm{mmol})$ and $\mathrm{CH}_{2} \mathrm{Cl}_{2}(8 \mathrm{~mL})$. After cooling to $-78^{\circ} \mathrm{C}$, dimethyl sulfoxide $(0.387 \mathrm{~mL}, 5.46 \mathrm{mmol})$

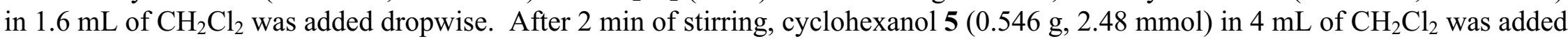
over $5 \mathrm{~min}$. The reaction mixture was stirred at $-78^{\circ} \mathrm{C}$ for $15 \mathrm{~min}$. Triethylamine $(1.73 \mathrm{~mL}, 12.4 \mathrm{mmol})$ was then added and the resulting mixture was stirred for an additional $5 \mathrm{~min}$ at $-78^{\circ} \mathrm{C}$, followed by warming to r.t. slowly. Water $(20 \mathrm{~mL})$ was added to the reaction mixture and product was extracted with $20 \mathrm{~mL}$ of $\mathrm{CH}_{2} \mathrm{Cl}_{2}$. The combined organic layer was washed sequentially with $1 \%$ $\mathrm{HCl}(1 \times 50 \mathrm{~mL})$, saturated $\mathrm{NaHCO}_{3}(1 \times 50 \mathrm{~mL})$, and brine. After drying over $\mathrm{MgSO}_{4}$ and evaporation of solvent, the crude ketone was purified by column chromatography $\left(10 \%\right.$ EtOAc/hexanes) to give $0.530 \mathrm{~g}(98 \%)$ of 6 as a light yellow oil. ${ }^{1} \mathrm{H}$ NMR: $\delta 1.59-1.81$ $(\mathrm{m}, 2 \mathrm{H}), 1.99-2.04(\mathrm{~m}, 2 \mathrm{H}), 2.10-2.25(\mathrm{~m}, 2 \mathrm{H}), 2.28(\mathrm{~s}, 3 \mathrm{H}), 2.49-2.53(\mathrm{~m}, 2 \mathrm{H}), 3.74(\mathrm{~s}, 3 \mathrm{H}), 3.89(\mathrm{dd}, 1 \mathrm{H}, \mathrm{J}=12.7,5.4 \mathrm{~Hz}), 6.77(\mathrm{~d}$, $1 \mathrm{H}, \mathrm{J}=8.3 \mathrm{~Hz}), 6.91(\mathrm{~d}, 1 \mathrm{H}, \mathrm{J}=2.0 \mathrm{~Hz}), 7.03(\mathrm{dd}, 1 \mathrm{H}, \mathrm{J}=8.2,2.0 \mathrm{~Hz}) .{ }^{13} \mathrm{C} \mathrm{NMR}: \delta 20.57,25.58,27.39,33.29,42.18,50.94,55.49$, $110.46,127.51,128.17,129.42,129.48,154.71,210.02$. IR (neat): 2935, 2861, 1714, 1612, 1589, 1504, 1449, 1354, 1317, 1289, $1248,1197,1126,1066,1034,882,838,806,732,706 \mathrm{~cm}^{-1}$. EI HRMS: calculated for $\mathrm{C}_{14} \mathrm{H}_{18} \mathrm{O}_{2} 218.1307$, found 218.1310 .

2-(2-Methoxy-5-methylphenyl)-2-methylcyclohexanone (6a). In a $25 \mathrm{~mL}$ round bottom flask equipped with a magnetic stirring bar, sodium hydride $(0.110 \mathrm{~g}, 4.58 \mathrm{mmol})$ was added to $5 \mathrm{~mL}$ of dimethoxyethane (DME). Cyclohexanone 6 (1.00g, $4.59 \mathrm{mmol})$ in $5 \mathrm{~mL}$ of DME was then added dropwise with stirring. The mixture was refluxed for $1 \mathrm{~h}$. Methyl iodide $(0.517 \mathrm{~mL}, 9.17 \mathrm{mmol}) \mathrm{was}$ added and the reaction mixture was allowed to reflux for $1 \mathrm{~h}$. After cooling to r.t., the mixture was poured into $5 \mathrm{~mL}$ of water and the product was extracted with ether $(3 \times 10 \mathrm{~mL})$. The combined organic layer was washed with brine, dried over $\mathrm{MgSO}_{4}$ and concentrated. Purification by column chromatography (15\% EtOAc/hexanes) followed by recrystallization from hexanes afforded $0.227 \mathrm{~g}$ of unreacted 6 and $0.515 \mathrm{~g}\left(63 \%\right.$, based on recovered starting material) of $\mathbf{6 a}$ as a crystalline solid. m.p. 82-83 ${ }^{\circ} \mathrm{C} .{ }^{1} \mathrm{H} \mathrm{NMR:} \delta 1.28(\mathrm{~s}$, $3 \mathrm{H}), 1.55-1.65(\mathrm{~m}, 2 \mathrm{H}), 1.70-1.80(\mathrm{~m}, 2 \mathrm{H}), 1.90-2.00(\mathrm{~m}, 1 \mathrm{H}), 2.20-2.30(\mathrm{~m}, 1 \mathrm{H}), 2.33(\mathrm{~s}, 3 \mathrm{H}), 2.38-2.50(\mathrm{~m}, 1 \mathrm{H}), 2.52-2.60(\mathrm{~m}, 1 \mathrm{H})$, 
$3.69(\mathrm{~s}, 3 \mathrm{H}), 6.77(\mathrm{~d}, \mathrm{~J}=8.2 \mathrm{~Hz}), 7.03(\mathrm{dd}, \mathrm{J}=8.2,2.0 \mathrm{~Hz}), 7.13(\mathrm{~d}, \mathrm{~J}=2.0 \mathrm{~Hz}) .{ }^{13} \mathrm{C}$ NMR: $\delta$ 20.77, 21.75, 24.57, 28.65, 39.10, 39.98, 51.96, 55.13, 111.52, 127.16, 128.00, 130.00, 133.14, 154.30, 214.52. IR (KBr): 2963, 2938, 2863, 2834, 1704, 1505, 1465, 1456, 1442, 1292, 1248, 1235, 1027, 810, 773, $708 \mathrm{~cm}^{-1}$. EI HRMS: calculated for $\mathrm{C}_{15} \mathrm{H}_{20} \mathrm{O}_{2} 232.1463$, found 232.1468 .

2-(2-Methoxy-5-methylphenyl)-2,6-dimethylcyclohexanone (7). A $50 \mathrm{~mL}$ round bottom flask equipped with a magnetic stirring bar was charged with diisopropylamine $(0.542 \mathrm{~mL}, 3.86 \mathrm{mmol})$ and $\mathrm{THF}(5 \mathrm{~mL})$. After cooling to $-78^{\circ} \mathrm{C}, n$-butyllithium $(3.76 \mathrm{~mL}, 3.69$ $\mathrm{mmol})$ was added dropwise, and the resulting mixture was stirred at $-78^{\circ} \mathrm{C}$ for $30 \mathrm{~min}$. Cyclohexanone $6 \mathrm{a}(0.815 \mathrm{~g}, 3.51 \mathrm{mmol})$ in 5 $\mathrm{mL}$ of THF was added dropwise, and reaction was allowed to come to r.t. After stirring at r.t. for $1 \mathrm{~h}$, reaction mixture was cooled to $0^{\circ} \mathrm{C}$, and methyl iodide $(0.241 \mathrm{~mL}, 3.86 \mathrm{mmol})$ was added dropwise. The reaction mixture was allowed to come to r.t. and was stirred for $18 \mathrm{~h}$ at r.t. Water $(10 \mathrm{~mL})$ was added and product was extracted with ether $(3 \times 10 \mathrm{~mL})$. The combined organic layer was washed with brine, dried over $\mathrm{MgSO}_{4}$, and concentrated. The crude product was purified by column chromatography (5\% EtOAc/hexanes) to give $7(0.821 \mathrm{~g}, 95 \%)$ as an amorphous solid and a mixture of diastereomers (diastereomeric ratio $\sim 11: 1)$. Spectroscopic data are given for major isomer: ${ }^{1} \mathrm{H}$ NMR: $\delta 0.97(\mathrm{~d}, 3 \mathrm{H}, \mathrm{J}=6.4 \mathrm{~Hz}), 1.22(\mathrm{~s}, 3 \mathrm{H}), 1.29-1.35(\mathrm{~m}, 1 \mathrm{H}), 1.50-1.60(\mathrm{~m}, 2 \mathrm{H}), 1.70-1.90(\mathrm{~m}, 2 \mathrm{H})$, $2.34(\mathrm{~s}, 3 \mathrm{H}), 2.45-2.55(\mathrm{~m}, 1 \mathrm{H}), 2.56-2.65(\mathrm{~m}, 1 \mathrm{H}), 3.66(\mathrm{~s}, 3 \mathrm{H}), 6.75(\mathrm{~d}, 1 \mathrm{H}, \mathrm{J}=8.2 \mathrm{~Hz}), 7.03(\mathrm{dd}, 1 \mathrm{H}, \mathrm{J}=8.2 \mathrm{~Hz}, 2.0 \mathrm{~Hz}), 7.16(\mathrm{~d}$, $1 \mathrm{H}, \mathrm{J}=2.0 \mathrm{~Hz}) .{ }^{13} \mathrm{C}$ NMR: $\delta 14.86,20.84,21.70,25.24,38.80,40.37,41.63,52.46,55.18,111.28,127.26,127.96,130.13,132.82$, 154.44, 215.98. IR (neat): 2966, 2930, 2861, 1715, 1500, 1458, 1369, 1292, 1251, 1238, 1031, $807 \mathrm{~cm}^{-1}$. EI HRMS: calculated for $\mathrm{C}_{16} \mathrm{H}_{22} \mathrm{O}_{2} 246.1620$, found 246.1626 .

Trans-3-(2-methoxy-5-methylphenyl)-1,3-dimethyl-2-oxocyclohexanecarboxylic acid methyl ester (3). In a $25 \mathrm{~mL}$ round bottom flask equipped with a magnetic stirring bar, diisopropylamine $(0.478 \mathrm{~mL}, 3.41 \mathrm{mmol})$ was added to $5 \mathrm{~mL}$ of ether. After cooling to $78^{\circ} \mathrm{C}$ in a dry ice-acetone bath, $n$-butyllithium $(3.13 \mathrm{mmol}, 2.08 \mathrm{~mL}, 1.5 \mathrm{M}$ in hexanes) was added dropwise. The reaction mixture was stirred at $-78^{\circ} \mathrm{C}$ for $1 \mathrm{~h}$. Cyclohexanone $7(0.700 \mathrm{~g}, 2.84 \mathrm{mmol})$ in $5 \mathrm{~mL}$ of ether was added dropwise. After stirring for an additional 30 min at $-78^{\circ} \mathrm{C}$, the reaction mixture was warmed to $0^{\circ} \mathrm{C}$ and stirred for $1 \mathrm{~h}$. The reaction mixture was then re-cooled to $-78^{\circ} \mathrm{C}$ and methyl cyanoformate $(0.316 \mathrm{~mL}, 3.98 \mathrm{mmol})$ was added dropwise. After stirring for $30 \mathrm{~min}$ at $-78^{\circ} \mathrm{C}$ and $18 \mathrm{~h}$ at r.t., saturated $\mathrm{NH}{ }_{4} \mathrm{Cl}$ $(10 \mathrm{~mL})$ was added and the product was extracted with ether $(3 \times 5 \mathrm{~mL})$. The combined organic layer was washed with brine, dried over $\mathrm{MgSO}_{4}$ and concentrated in vacuo. The residue was purified by flash chromatography $(10 \% \mathrm{EtOAc} /$ hexanes $)$ to give 3 (0.626 g, $72 \%)$ as a white crystalline solid. m.p. $96-97^{\circ} \mathrm{C} .{ }^{1} \mathrm{H}$ NMR: $\delta 1.40(\mathrm{~s}, 3 \mathrm{H}), 1.46(\mathrm{~s}, 3 \mathrm{H}), 1.60-1.70(\mathrm{~m}, 1 \mathrm{H}), 1.70-1.80(\mathrm{~m}, 1 \mathrm{H}), 1.80-$ $1.95(\mathrm{~m}, 2 \mathrm{H}), 2.22-2.30(\mathrm{~m}, 1 \mathrm{H}), 2.30(\mathrm{~s}, 3 \mathrm{H}), 2.45-2.55(\mathrm{~m}, 1 \mathrm{H}), 3.71(\mathrm{~s}, 3 \mathrm{H}), 3.74(\mathrm{~s}, 3 \mathrm{H}), 6.75(\mathrm{~d}, 1 \mathrm{H}, \mathrm{J}=8.2 \mathrm{~Hz}), 7.02(\mathrm{dd}, 1 \mathrm{H}, \mathrm{J}=$ 8.2 , $2.0 \mathrm{~Hz}), 7.09(\mathrm{~d}, 1 \mathrm{H}, \mathrm{J}=2.0 \mathrm{~Hz}){ }^{13} \mathrm{C}$ NMR: $\delta$ 19.47, 20.84, 23.75, 23.76, 36.11, 39.68, 51.24, 52.28, 55.06, 56.08, 111.17, 126.98, 127.94, 129.66, 135.32, 153.36, 174.05, 209.84. IR (KBr): 2975, 2948, 2921, 1732, 1711, 1498, 1463, 1450, 1240, 1206, 1185, 1132, 1030, 1006, 984, $816 \mathrm{~cm}^{-1}$. HRMS: Calculated for $\mathrm{C}_{18} \mathrm{H}_{24} \mathrm{O}_{4} 304.1675$, found 304.1673. 
Trans-2-(2-Methoxy-5-methylphenyl)-1,2-dimethylcyclopentanecarboxylic acid methyl ester (2). Ground powder of crystalline 3 (45 mg, $0.148 \mathrm{mmol}$ ) was placed in between two microscope slides and cooled with an ice bath. The sample was irradiated with a medium pressure Hanovia mercury arc lamp with a 310nm filter for $12 \mathrm{~h}$. Crude product was washed off with $\mathrm{CH}_{2} \mathrm{Cl}_{2}$ and purified by preparative tlc (5\% EtOAc/ hexanes). Ester 2 was obtained as a lightly yellow oil in $63 \%$ yield (8 $\mathrm{mg}, 0.029 \mathrm{mmol})$ based on unreacted starting material $(31 \mathrm{mg}, 0.102 \mathrm{mmol}) .{ }^{1} \mathrm{H}$ NMR: $\delta 0.85(\mathrm{~s}, 3 \mathrm{H}), 1.44(\mathrm{~s}, 3 \mathrm{H}), 1.65-2.00(\mathrm{~m}, 4 \mathrm{H}), 2.27(\mathrm{~s}, 3 \mathrm{H}), 2.40-2.52$ $(\mathrm{m}, 2 \mathrm{H}), 3.65(\mathrm{~s}, 3 \mathrm{H}), 3.73(\mathrm{~s}, 3 \mathrm{H}), 6.70(\mathrm{~d}, 1 \mathrm{H}, \mathrm{J}=8.2 \mathrm{~Hz}), 6.98(\mathrm{dd}, 1 \mathrm{H}, \mathrm{J}=8.2 \mathrm{~Hz}, 1.5 \mathrm{~Hz}), 7.03(\mathrm{~d}, 1 \mathrm{H}, \mathrm{J}=2.0 \mathrm{~Hz}) .13 \mathrm{C} \mathrm{NMR}: \delta$ 19.99, 20.80, 23.02, 25.15, 39.56, 51.10, 53.41, 53.61, 54.52, 110.51, 127.44, 128.86, 129.12, 135.23, 155.31, 178.06. IR (neat): 2946, 2927, 1725, 1500, 1462, 1376, 1293, 1254, 1178, 1158, 1090, 1032, 805, 758, $724 \mathrm{~cm}^{-1}$. EI HRMS: calculated for $\mathrm{C}_{17} \mathrm{H}_{24} \mathrm{O}_{3}$ 276.1725 , found 276.1729 .

Herbertenolide (1). Ester $2(8 \mathrm{mg}, 0.0290 \mathrm{mmol})$ was dissolved in $\mathrm{CH}_{2} \mathrm{Cl}_{2}(3 \mathrm{~mL})$ in a $25 \mathrm{~mL}$ round bottom flask equipped with a magnetic stirring bar and cooled to $0^{\circ} \mathrm{C}$. Boron tribromide $\left(0.200 \mathrm{~mL}, 1 \mathrm{M}\right.$ in $\left.\mathrm{CH}_{2} \mathrm{Cl}_{2}\right)$ was then added dropwise with stirring. After stirring for $1 \mathrm{~h}$, the mixture was allowed to come to r.t. and continued to stir for $18 \mathrm{~h}$. The reaction was quenched with saturated $\mathrm{NH}_{4} \mathrm{Cl}$ $(5 \mathrm{~mL})$ and the product was extracted with $\mathrm{CH}_{2} \mathrm{Cl}_{2}(2 \times 5 \mathrm{~mL})$. The combined organic layer was washed with brine, dried over $\mathrm{MgSO}_{4}$, and concentrated to give a brown residue. The crude lactone 1 was purified by preparative tlc (5\% EtOAc/hexanes) and $4 \mathrm{mg}$ $(60 \%)$ of 1 was obtained as a white solid (crystallized from ether). m.p. $52-53{ }^{\circ} \mathrm{C} .{ }^{1} \mathrm{H}$ NMR: $\delta 0.93(\mathrm{~s}, 3 \mathrm{H}), 1.13(\mathrm{~s}, 3 \mathrm{H}), 1.70-1.80(\mathrm{~m}$, $1 \mathrm{H}), 1.85-2.05(\mathrm{~m}, 4 \mathrm{H}), 2.15-2.25(\mathrm{~m}, 1 \mathrm{H}), 2.33(\mathrm{~s}, 3 \mathrm{H}), 6.89(\mathrm{~d}, 1 \mathrm{H}, \mathrm{J}=1.8 \mathrm{~Hz}), 6.93(\mathrm{~d}, 1 \mathrm{H}, \mathrm{J}=8.2 \mathrm{~Hz}), 7.02(\mathrm{dd}, 1 \mathrm{H}, \mathrm{J}=8.2 \mathrm{~Hz}$, $1.8 \mathrm{~Hz}) .{ }^{13} \mathrm{C}$ NMR: $19.78,19.88,20.89,25.35,29.03,29.65,47.07,50.80,115.82,125.28,128.02,133.02,134.06,149.62,173.60$. IR (film): 2967, 2926, 1777, 1489, 1460, 1445, 1377, 1267, 1206, 1192, 1082, 1047, 928, $816 \mathrm{~cm}^{-1}$. EI HRMS: calculated for $\mathrm{C}_{15} \mathrm{H}_{18} \mathrm{O}_{2} 230.1307$, found 230.1308. 


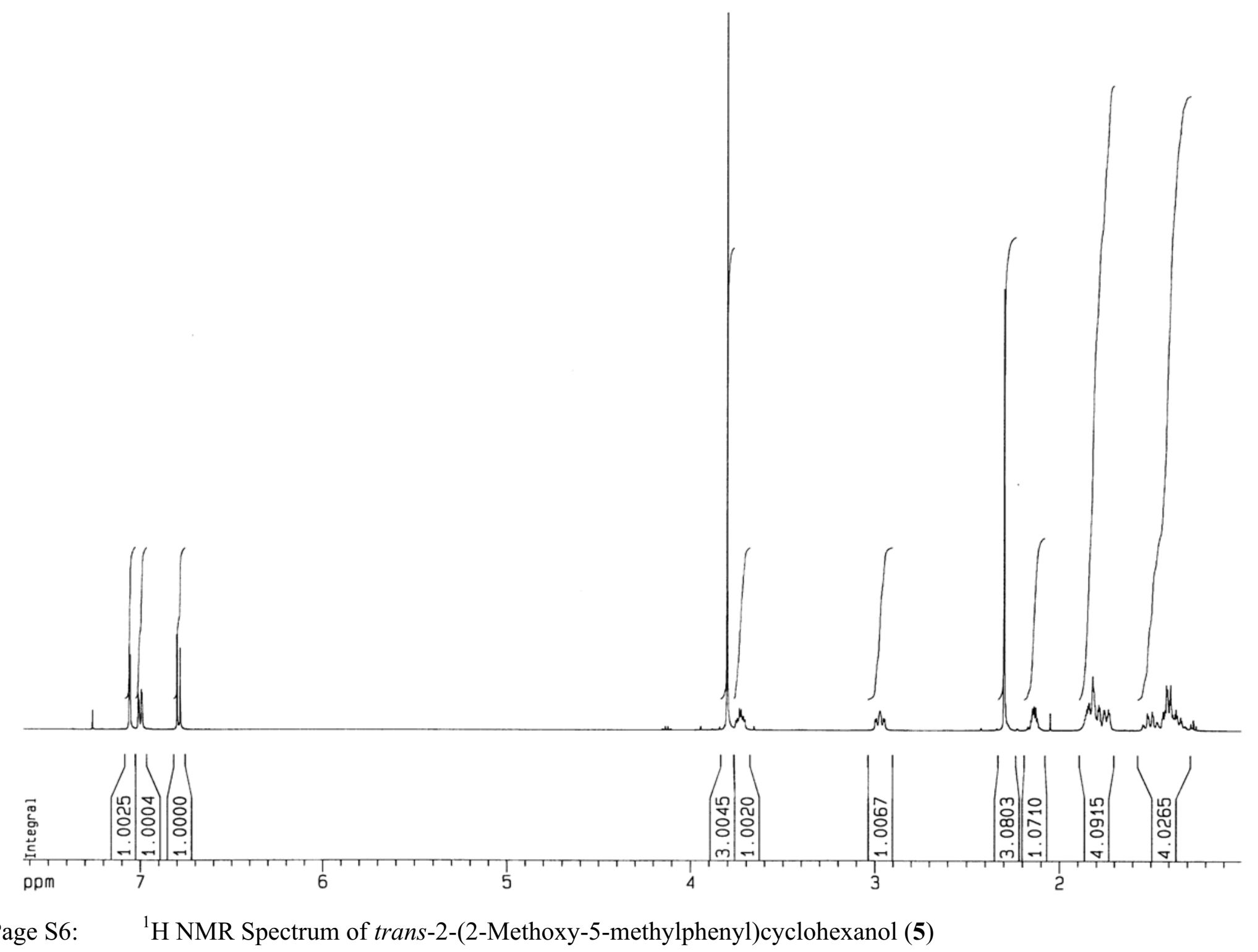

Page S6 


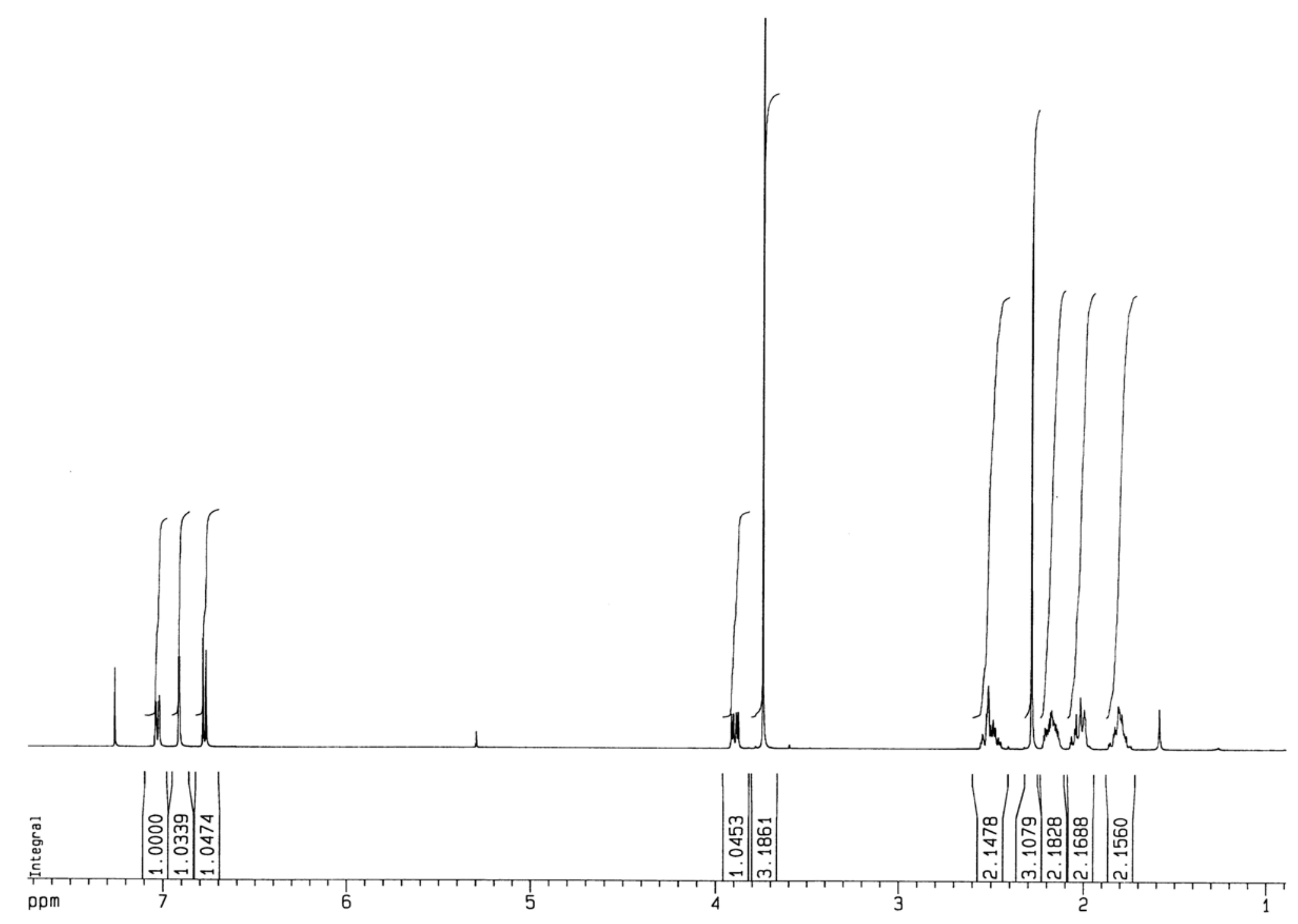

Page S7: ${ }^{1}$ H NMR Spectrum of 2-(2-Methoxy-5-methylphenyl)cyclohexanone (6)

Page S7 


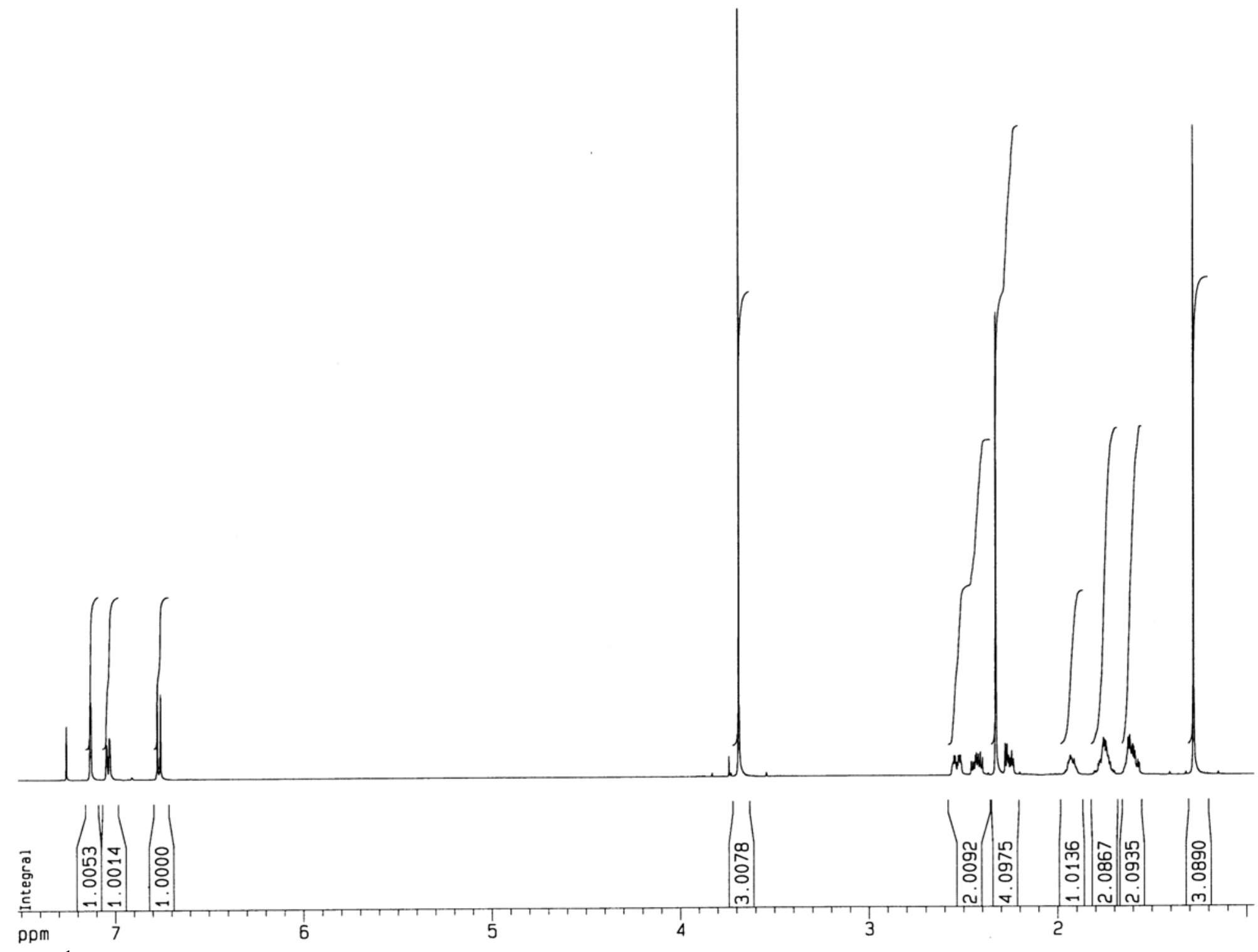

Page S8: ${ }^{1} \mathrm{H}$ NMR Spectrum of 2-(2-Methoxy-5-methylphenyl)-2-methylcyclohexanone (6a)

Page S8 


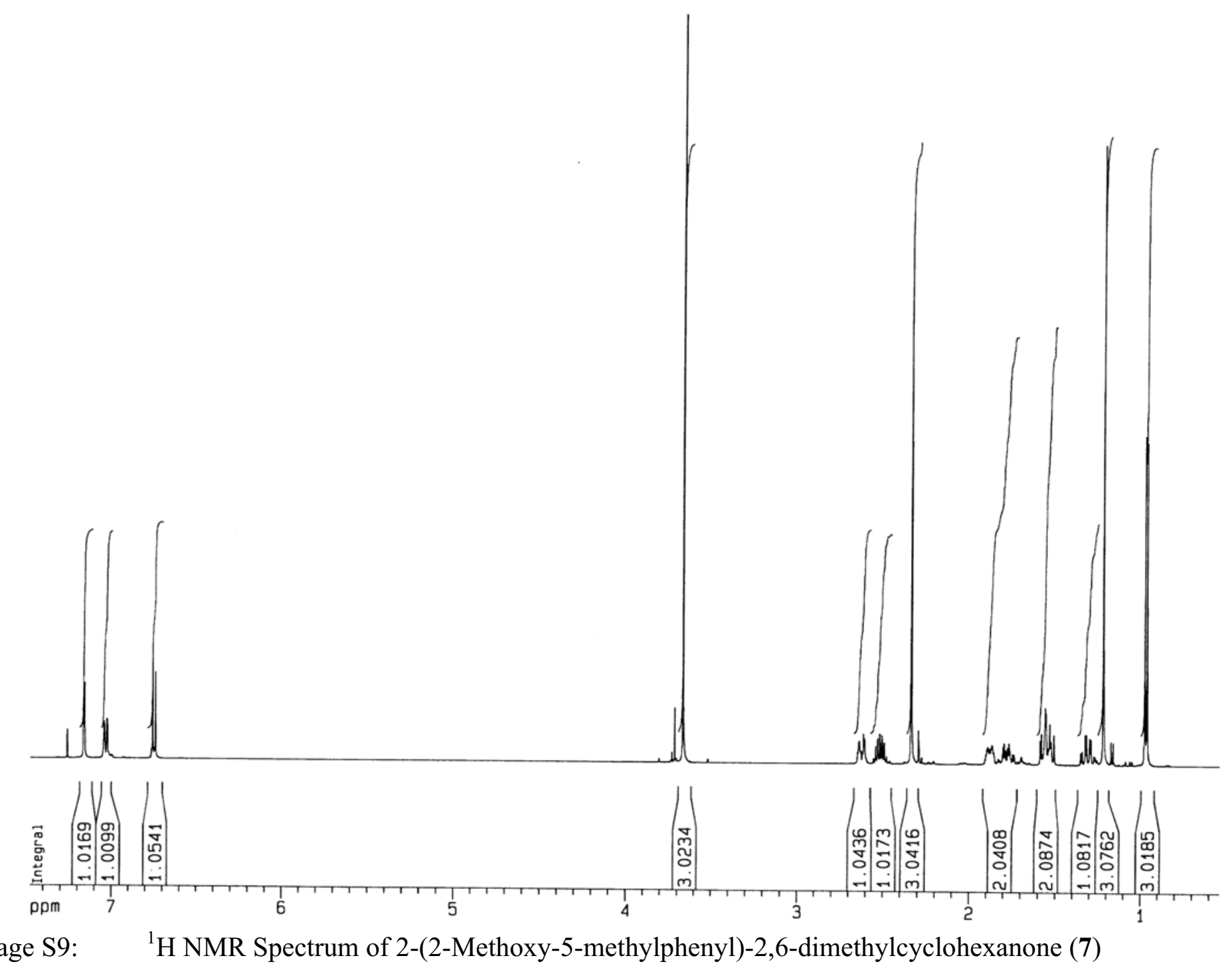

Page S9 


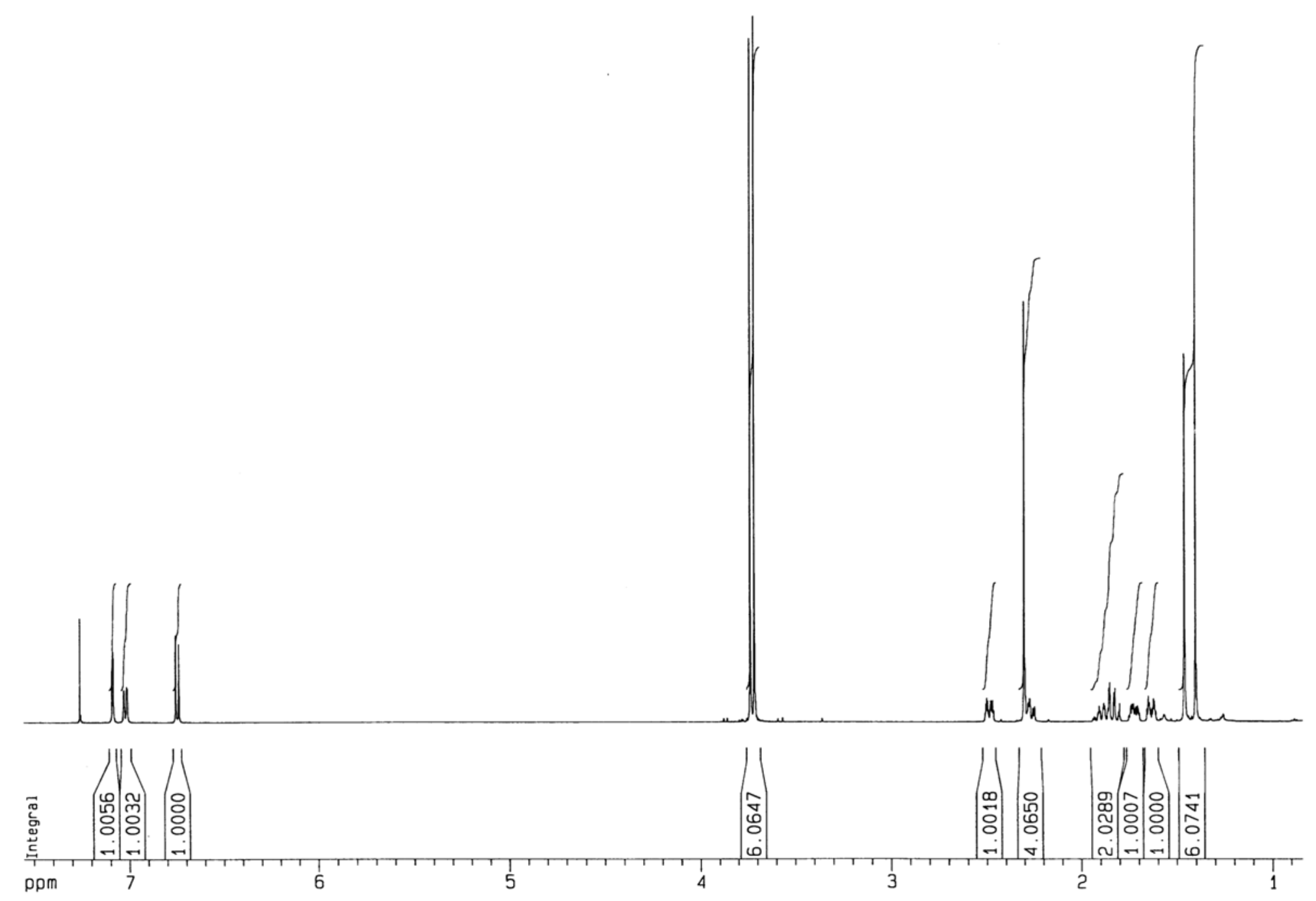

Page S10: ${ }^{1} \mathrm{H}$ NMR Spectrum of trans-3-(2-Methoxy-5-methylphenyl)-1,3-dimethyl-2-oxocyclohexanecarboxylic acid methyl ester (3) 


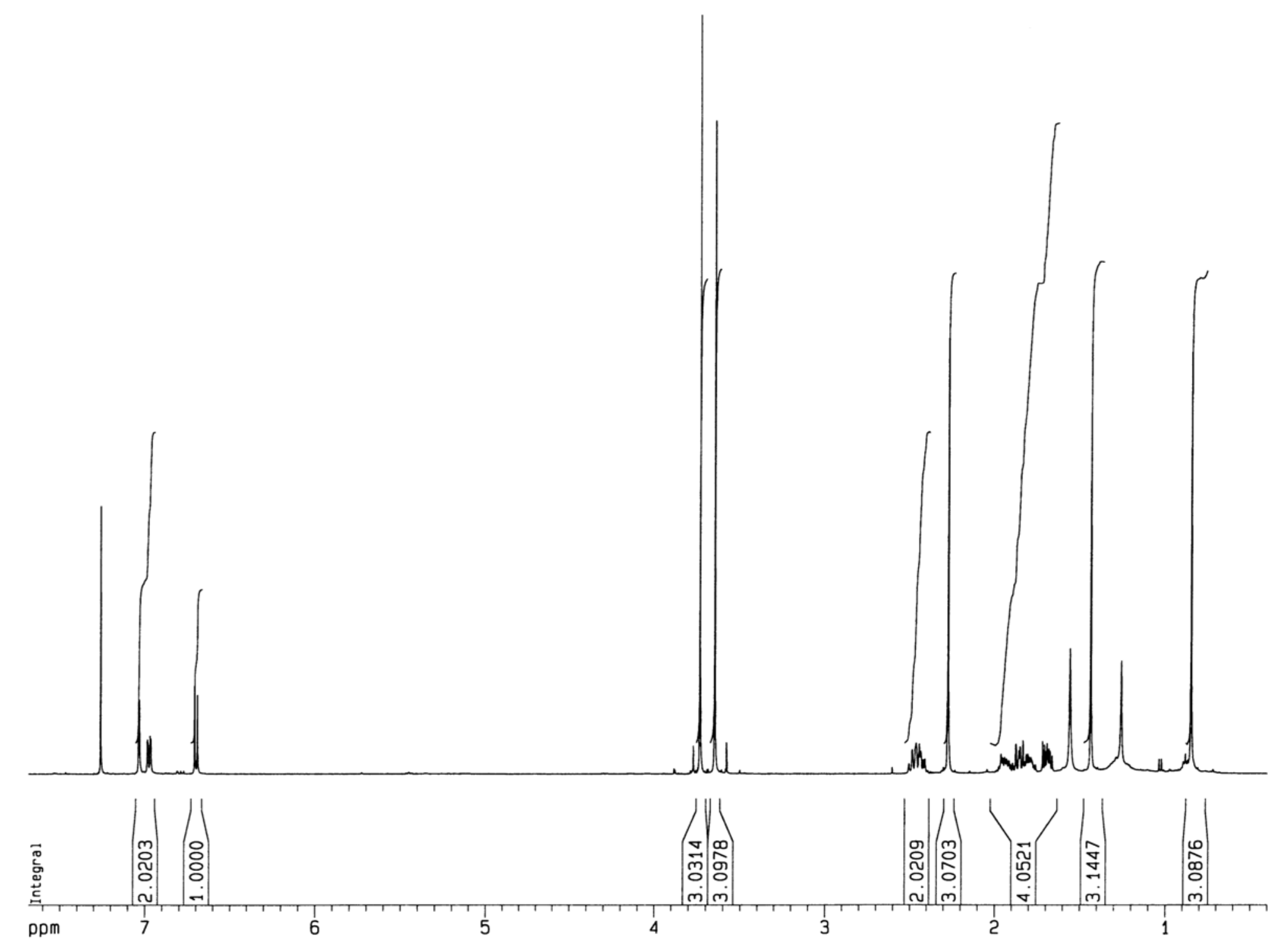

Page S11: ${ }^{1} \mathrm{H}$ NMR Spectrum of trans-2-(2-Methoxy-5-methylphenyl)-1,2-dimethylcyclopentanecarboxylic acid methyl ester (2) 


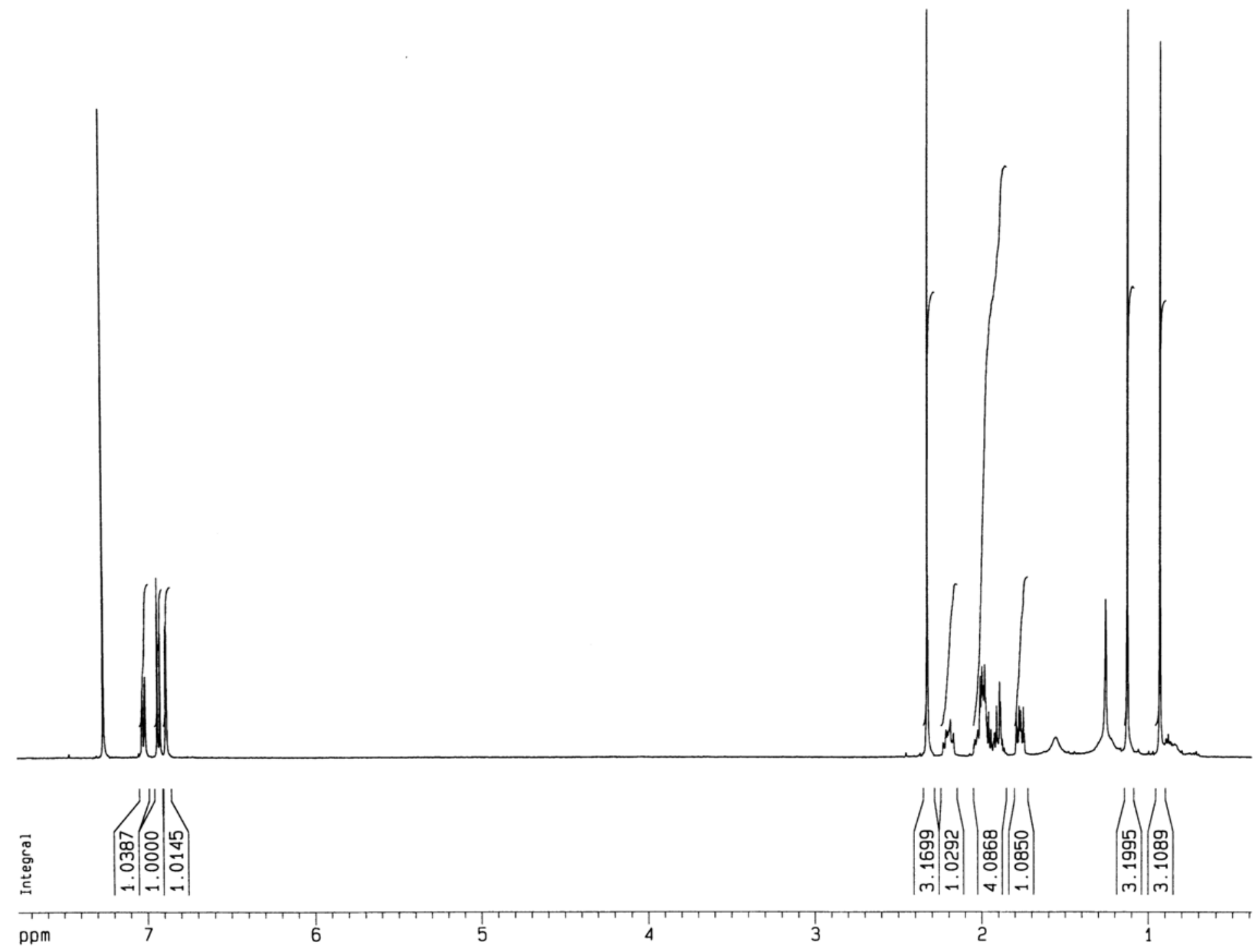

Page S12: ${ }^{1} \mathrm{H}$ NMR Spectrum of $( \pm)$-herbertenolide (1). 